\title{
Non-Markovian homodyne-mediated feedback on a two-level atom: a quantum trajectory treatment
}

\author{
Jin Wang ${ }^{1}$, H.M. Wiseman ${ }^{2}$, G.J. Milburn ${ }^{3}$ \\ ${ }^{1}$ Centre for Laser Science, Department of Physics, The University of Queensland, Brisbane, Queensland 4072, Australia \\ 2 School of Science, Griffith University, Brisbane, Queensland 4111, Australia \\ ${ }^{3}$ Centre for Quantum Computer Technology, The University of Queensland, Brisbane, Queensland 4072, Australia
}

(February 1, 2008)

\begin{abstract}
Quantum feedback can stabilize a two-level atom against decoherence (spontaneous emission), putting it into an arbitrary (specified) pure state. This requires perfect homodyne detection of the atomic emission, and instantaneous feedback. Inefficient detection was considered previously by two of us. Here we allow for a non-zero delay time $\tau$ in the feedback circuit. Because a two-level atom is a nonlinear optical system, an analytical solution is not possible. However, quantum trajectories allow a simple numerical simulation of the resulting non-Markovian process. We find the effect of the time delay to be qualitatively similar to that of inefficient detection. The solution of the non-Markovian quantum trajectory will not remain fixed, so that the time-averaged state will be mixed, not pure. In the case where one tries to stabilize the atom in the excited state, an approximate analytical solution to the quantum trajectory is possible. The result, that the purity $\left(P=2 \operatorname{Tr}\left[\rho^{2}\right]-1\right)$ of the average state is given by $P=1-4 \gamma \tau$ (where $\gamma$ is the spontaneous emission rate) is found to agree very well with the numerical results.
\end{abstract}

42.50.Lc, 42.50.Ct, 03.65.Bz

\section{INTRODUCTION}

The theory of open quantum systems is fundamental to understanding dissipation on a microscopic and macroscopic level in diverse fields (measurement theory, quantum optics, quantum chaos, solid state, quantum computation) [1] 5], wherever quantum irreversibility matters. Traditionally, open quantum systems are described by the reduced density operator, which is obtained from the total density operator by tracing over the environmental degrees of freedom. The dynamics of the reduced density operator is commonly described by a master equation.

Besides the density matrix formalism, there has been an increasing interest over the last years in the quantum trajectory approach which uses a stochastic Schrödinger equation for the state vector $|\psi\rangle$ [6] [8]. This approach serves as a numerical tool for solving the master equation, as the reduced density operator is recovered as the ensemble average of these stochastically evolving pure states. It also has a deeper significance, as, in some cases, the quantum trajectories can be interpreted as the evolution of the system state conditioned on continuous monitoring of its environment [9, 10]. This means that quantum trajectories are ideal for treating quantum feedback, where the measurement results from this monitoring are used to control the dynamics of the system [11,12].

The history of feedback-control in open quantum systems goes back to 1980's with the work of Yamamoto and co-workers 113,14], and Shapiro and co-workers [15]. Their objective was to explain the observation [16] of subshot-noise fluctuations in an in-loop photocurrent. They did this using quantum Langevin equations (stochastic Heisenberg equations for the system operators) and also semiclassical techniques. The latter approach was made fully quantum-mechanical by Plimak 17]. For systems with linear dynamics, all of these approaches, and the quantum trajectory approach of Refs. [11,12], are equally easy to use to find analytical solutions. The advantage of the Wiseman and Milburn (quantum trajectory) approach to quantum control via feedback is for systems with nonlinear dynamics, as we will discuss later.

Before proceeding further, it is necessary to distinguish what we mean by 'quantum control' via feedback from an alternative usage of the term 'quantum control' which has become current in laser chemistry [18]. The work in this field has sought sufficient control over laser pulses so that very specific quantum dynamics of electronic and vibrational states may be achieved, primarily with the objective of making and breaking bonds in molecular systems. Typically such experiments are conducted on a large ensemble of identical prepared target systems. Two kinds of control are distinguished; (i) learning control, and, (ii) feedback control. In learning control the objective is to design the required laser pulse as follows. After applying a pulse to a sample of many identical constituents (an ensemble), one measures some property of the system, adjusts the laser pulse according to the measurement results, then applies the new laser pulse to a new sample and so on. The cycle of adjust-excite-measure is repeated until the desired results are obtained. In feedback control, the laser excitation always acts on the same sample of many identical systems without re-preparation, through the same repeated sequence. Of course the adjustment one makes to the next pulse will be quite different in the two cases.

In contrast to this controlling of the average state of an ensemble, we are concerned with the control of the state of a single quantum system subject to continuous 
measurement. While this is similar to (ii), the fact that only a single quantum system is involved, not an ensemble, makes it fundamentally different. The quantum efficiency of the measurement has to be relatively large for the control to be at all effective. This means that one has to take into account the quantum back-action of the measurement on the system, which is not necessary in the laser chemistry 'feedback control' referred to above. Only very recently has it become possible to isolate, and continuously interrogate a single quantum system at the back-action limit. This has been achieved in a number of fields including cavity QED [19] ion trapping [20] and mesoscopic electronics 21.

Returning to the different approaches to control of a single quantum system, another advantage of the Wiseman-Milburn quantum feedback theory is that it is very easy to consider the limit of Markovian (i.e. instantaneous) feedback. In this limit it is possible to derive a master equation which describes the unconditioned system dynamics including the effect of feedback [11,12]. In previous work, two of us 22 applied Markovian quantum feedback theory to a two-level atom, where the light emitted by the atom was detected and used to control the dynamics of the atom. Using the feedback master equation, analytical results are easily derived. We showed that, with one exception, the feedback can generate an arbitrary stable pure state of the atom. This is an example of using feedback to control decoherence (due to spontaneous emission).

Decoherence in open quantum systems refers to the tendency for pure system states to become entangled with many different states of the environment 23. If the states of the environment are then averaged out, the system state tends to become mixed. The radiative decay of a two level atom with a non-zero dipole is an example. In this case the atomic state becomes correlated with the electromagnetic field states.

Our previous work 22] was motivated by the 1998 work of Hofmann, Mahler, and Hess (HMH) 24, in which it was shown that by making part of the coherent driving of a two-level atom proportional to the homodyne photocurrent, it was possible to stabilize the state at any point on the bottom half of the Bloch sphere. Our work [22], using the Wiseman-Milburn feedback theory, reanalyzed their proposal and generalized their results in two ways. First, we showed that any point on the upper or lower half (but not the equator) of the Bloch sphere may be stabilized. Second, we considered non-unit-efficiency detection, and quantified the effectiveness of the feedback by calculating the maximal purity obtainable in any particular direction in Bloch space.

It is now of interest to extend our theory of feedbackmediated decoherence control to a non-Markovian process. This would arise naturally in an experiment, since any feedback apparatus would necessarily have some delay associated with it. It is also of interest theoretically because non-Markovian feedback in a nonlinear system has not been considered in detail before. The two-level atom, being the simplest possible system with nonlinear dynamics, is an ideal testing ground for descriptions of non-Markovian processes. Of course the master equations derived in Refs. [11,12 cannot be applied to nonMarkovian feedback in which the delay is significant on the time scale of the system.

Most of previous approaches to non-Markovian feedback have been limited to linear systems such as an optical cavity mode with feedback based on homodynedetection. Wiseman and Milburn [25] used a quantum trajectory treatment, as did Doherty and Jacobs [26]. Giovannetti, Tombesi and Vitali [27] used the quantum Langevin approach of input-output theory. As noted above, linear systems allow analytical solutions, and all approaches to quantum feedback work equally as well. However, for a nonlinear system like a two-level atom an analytical solution for non-Markovian feedback is not possible in general. Hence a numerical solution is necessary. The only practical way of doing such a simulation is to use the stochastic quantum trajectories for the conditioned system state, which underly the WisemanMilburn feedback theory. Such a numerical quantum trajectory simulation was done recently for a non-linear system consisting of a particle in a double-well potential with feedback determined by LQG (linearized quadratic Gaussian) control theory 28].

Quantum trajectories describing non-Markovian feedback are of some intrinsic interest as they are a special example of non-Markovian quantum trajectories. These have been the subject of much interest lately. An example of this is the stochastic wave function method developed by Jack, Collett and Walls [29], where the non-Markovian stochastic equation of motion for the state vector involves a multiple time integration over the system's history conditioned on the measurement record over a finite time interval. Furthermore, it has been shown recently by Diosi and Strunz and co-workers [30,31 that it is in principle possible to construct a stochastic Schrödinger equation which describes the nonMarkovian time evolution of any open quantum system.

Using non-Markovian quantum trajectory simulations we are able to determine the effect of a time delay on the feedback stabilization scheme introduced in Refs. 22, 22, We find that the effect of a time delay is qualitatively similar to that of inefficient detectors. That is, the average state can no longer be made pure. States in the upper half of the Bloch sphere are more affected than those in the lower half, and states near the equator are affected most of all. In one special case, where the feedback aims to stabilize the atom in the excited state, it is possible to linearize the quantum trajectory if the time delay is short, and so obtain an approximate analytical solution. The result compares very well with the numerical solution. It is not obvious that this linearization could be performed using any of the other approaches to quantum feedback.

The paper is organized as following. In Sec. II we introduce the measurement scheme (homodyne detection) for 
our system (the two-level atom). In Sec. III, we briefly review our previous work of Markovian feedback stabilization. The effectiveness of non-Markovian feedback is studied in Sec. IV, both numerically and analytically. In Sec. $\mathrm{V}$ we conclude with a discussion of our results.

\section{THE MEASUREMENT SCHEME}

\section{A. The driven, damped atom}

Consider an atom, with two relevant levels $\{|g\rangle,|e\rangle\}$ and lowering operator $\sigma=|g\rangle\langle e|$. Let the decay rate be $\gamma$, and let it be driven by a resonant classical driving field with Rabi frequency $2 \alpha$. This is as shown in Fig. 1, where for the moment we are omitting feedback by setting $\lambda=0$. This system is well-approximated by the master equation

$$
\dot{\rho}=\gamma \mathcal{D}[\sigma] \rho-i \alpha\left[\sigma_{y}, \rho\right]
$$

where the Lindblad 32 superoperator is defined as usual $\mathcal{D}[A] B \equiv A B A^{\dagger}-\left\{A^{\dagger} A, B\right\} / 2$. In this master equation we have chosen to define the $\sigma_{x}=\sigma+\sigma^{\dagger}$ and $\sigma_{y}=i \sigma-i \sigma^{\dagger}$ quadratures of the atomic dipole relative to the driving field. The effect of driving is to rotate the atom in Bloch space around the $y$-axis. The state of the atom in Bloch space is described by the three-vector $(x, y, z)$. It is related to the state matrix $\rho$ by

$$
\rho=\frac{1}{2}\left(I+x \sigma_{x}+y \sigma_{y}+z \sigma_{z}\right) .
$$

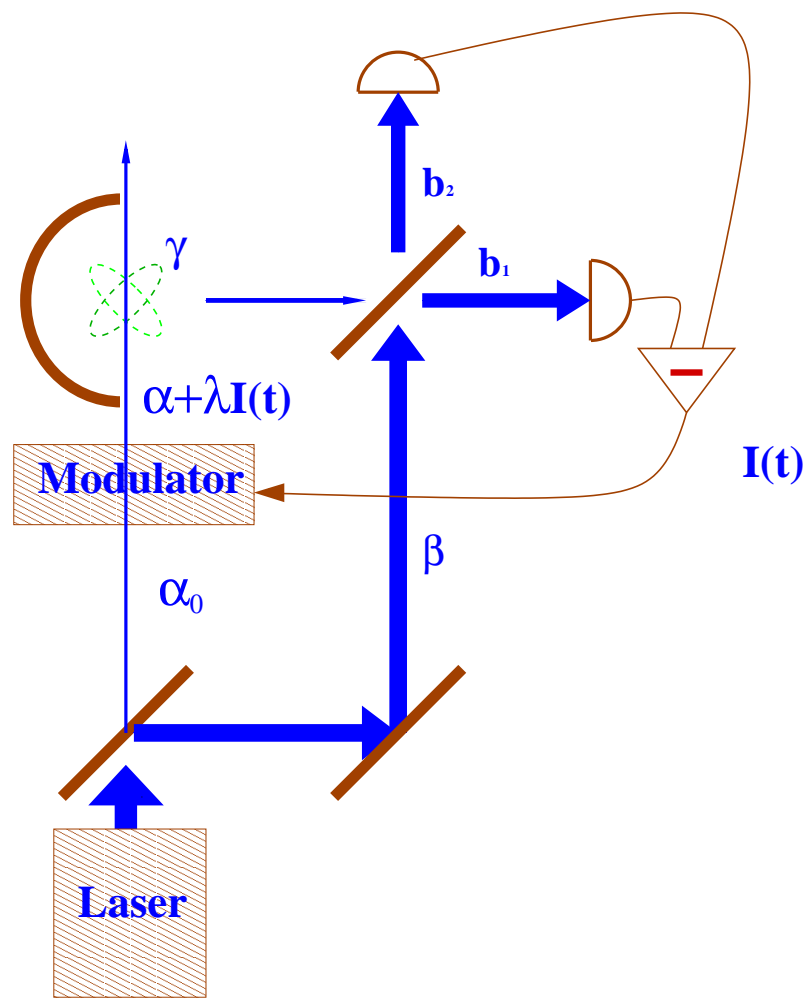

FIG. 1. Diagram of the experimental apparatus. The laser beam is split to produce both the local oscillator $\beta$ and the field $\alpha_{0}$ which is modulated using the current $I(t)$. The modulated beam, with amplitude proportional to $\alpha+\lambda I(t)$, drives an atom at the center of the parabolic mirror. The fluorescence thus collected is subject to homodyne detection using the local oscillator, and gives rise to the homodyne photocurrent $I(t)$.

It is easy to show that the stationary solution of the master equation (2.1) is

$$
\begin{aligned}
& x_{\mathrm{ss}}=\frac{-4 \alpha \gamma}{\gamma^{2}+8 \alpha^{2}}, \\
& y_{\mathrm{ss}}=0 \\
& z_{\mathrm{ss}}=\frac{-\gamma^{2}}{\gamma^{2}+8 \alpha^{2}} .
\end{aligned}
$$

For $\gamma$ fixed, this is a family of solutions parameterized by the driving strength $\alpha \in(-\infty, \infty)$. All members of the family are in the $x-z$ plane on the Bloch sphere. Thus for this purpose we can reparametrize the relevant states using $r$ and $\theta$ by

$$
x=r \sin \theta, \quad z=r \cos \theta,
$$

where $\theta \in[-\pi, \pi]$. Since

$$
\operatorname{Tr}\left[\rho^{2}\right]=\frac{1}{2}\left(1+x^{2}+y^{2}+z^{2}\right)
$$

is a measure of the purity of the Bloch sphere, $r=$ $\sqrt{x^{2}+z^{2}}$, the distance from the center of the sphere, is also a measure of purity. Pure states correspond to $r=1$ and maximally mixed states to $r=0$.

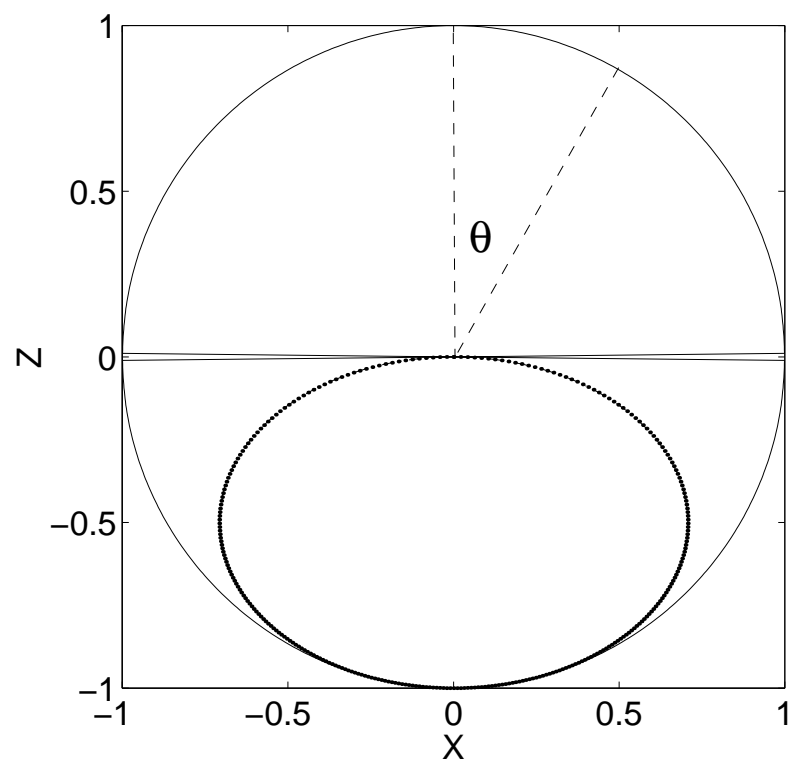

FIG. 2. Locus of the solutions to the Bloch equations. The ellipse in the lower half plane is the locus for the equations with driving only. The full circle (minus the points on the equator) is the locus for the equations with optimal driving and feedback, as defined in Sec. III. 
The locus of solutions in this plane (an ellipse) is shown in Fig. 2. Since $z_{\mathrm{ss}}<0$, all solutions are in the lower half of the Bloch sphere. That is, we are restricted to $|\theta|>\pi / 2$. Also, it is evident that the smaller $|\theta|$ is (that is, the more excited the atom is), the smaller $r$ is (that is, the less pure the atom is). At $|\theta|=\pi$ the stationary state is pure, but this is not surprising as it is simply the ground state of the atom with no driving. As $|\theta| \rightarrow \pi / 2$ we have $r \rightarrow 0$. This can only be approached asymptotically as $|\alpha| \rightarrow \infty$. In summary, the stationary states we can reach by driving the atom are limited, and generally far from pure.

\section{B. Homodyne detection}

Now consider subjecting the atom to homodyne detection. As shown in Fig. 11, we assume that all of the fluorescence of the atom is collected and turned into a beam (represented in Fig. 11 by placing the atom at the focus of a mirror). Ignoring the vacuum fluctuations in the field, the annihilation operator for this beam is $\sqrt{\gamma} \sigma$, normalized so that the mean intensity $\gamma\left\langle\sigma^{\dagger} \sigma\right\rangle$ is equal to the number of photons per unit time in the beam. This beam then enters one port of a 50:50 beam splitter, while a strong local oscillator $\beta$ enters the other. To ensure that this local oscillator has a fixed phase relationship with the driving laser used in the measurement, it would be natural to utilize the same coherent light field source in the driving process and as the local oscillator in the homodyne detection. This is as shown in Fig. 1.

Again ignoring vacuum fluctuations, the two field operators exiting the beam splitter, $b_{1}$ and $b_{2}$, are

$$
b_{k}=\left[\sqrt{\gamma} \sigma-(-1)^{k} \beta\right] / \sqrt{2} \text {. }
$$

When these two fields are detected, the two photocurrents produced have means

$$
\bar{I}_{k}=\left\langle|\beta|^{2}-(-1)^{k}\left(\sqrt{\gamma} \beta \sigma^{\dagger}+\sqrt{\gamma} \sigma \beta^{*}\right)+\gamma \sigma^{\dagger} \sigma\right\rangle / 2 .
$$

The middle two terms represent the interference between the system and the local oscillator.

Equation Eq. (2.9) gives only the mean photocurrent. In an individual run of the experiment for a system, what is recorded is not the mean photocurrent, but the instantaneous photocurrent. This photocurrent will vary stochastically from one run to the next, because of the irreducible randomness in the quantum measurement process. This randomness is not just noise, however. It is correlated with the evolution of the system and thus tells the experimenter something about the state of the system. In fact, if the detection efficiency is perfect, the system is collapsed into a pure state, rather than the mixed state which is the solution of the master equation. The stochastic evolution of the state of the system conditioned on the measurement record is called a "quantum trajectory" [6]. Of course, the master equation is still obeyed on average, so the set of possible quantum trajectories is called an unraveling of the master equation [6]. It is the conditioning of the system state on the photocurrent record that allows feedback of the photocurrent to control the system state. The application of an appropriate feedback loop to this continuous measurement process (to be considered in Sec. III) realizes an effective "reservoir engineering" to control the system at the quantum level.

The ideal limit of homodyne detection is when the local oscillator amplitude goes to infinity, which in practical terms means $|\beta|^{2} \gg \gamma$. In this limit, the rate of the photodetections goes to infinity and thus it should be possible to change the point process of photocounts into a continuous photocurrent with white noise. Also, the only relevant quantity is the difference between the two photocurrents. Suitably normalized, this is [6, 33]

$$
I(t)=\frac{I_{1}(t)-I_{2}(t)}{|\beta|}=\sqrt{\gamma}\left\langle e^{-i \Phi} \sigma^{\dagger}+e^{i \Phi} \sigma\right\rangle_{\mathrm{c}}(t)+\xi(t) .
$$

A number of aspects of Eq. (2.10) need to be explained. First, $\Phi=\arg \beta$, the phase of the local oscillator (defined relative to the driving field). Second, the subscript c means conditioned and refers to the fact that if one is making a homodyne measurement then this yields information about the system. Hence, any system averages will be conditioned on the previous photocurrent record. Third, the final term $\xi(t)$ represents Gaussian white noise, so that

$$
\xi(t) d t=d W(t)
$$

an infinitesimal Wiener increment defined by 34

$$
\begin{gathered}
d W(t)^{2}=d t \\
\mathrm{E}[d W(t)]=0 .
\end{gathered}
$$

Since the stationary solution of the master equation confines the state to the $x-z$ plane, it makes sense to follow $\mathrm{HMH}$ by setting $\Phi=0$. In that case,

$$
I(t)=\sqrt{\gamma}\left\langle\sigma_{x}\right\rangle_{\mathrm{c}}(t)+\xi(t) .
$$

That is, the deterministic part of the homodyne photocurrent is proportional to $x_{\mathrm{c}}=\left\langle\sigma_{x}\right\rangle_{\mathrm{c}}$. This should be useful for controlling the dynamics of the state in the $x-z$ plane by feedback, as we will consider in Sec. III. Of course, all that really matters here is the relationship between the driving phase and the local oscillator phase, not the absolute phase of either.

The conditioning process referred to above can be made explicit by calculating how the system state changes in response to the measured photocurrent. Assuming that the state at some point in time is pure (which will tend to happen because of the conditioning anyway), its future evolution can be described by the stochastic Schrödinger equation SSE. [6, 33] 


$$
d\left|\psi_{\mathrm{c}}(t)\right\rangle=\hat{A}_{\mathrm{c}}(t)\left|\psi_{\mathrm{c}}(t)\right\rangle d t+\hat{B}_{\mathrm{c}}(t)\left|\psi_{\mathrm{c}}(t)\right\rangle d W(t) .
$$

This is an Itô stochastic equation 34 with a drift term and a diffusion term. The operator for the drift term is

$$
\hat{A}_{\mathrm{c}}(t)=\frac{\gamma}{2}\left[-\sigma^{\dagger} \sigma+\left\langle\sigma_{x}\right\rangle_{\mathrm{c}}(t) \sigma-\left\langle\sigma_{x}\right\rangle_{\mathrm{c}}^{2}(t) / 4\right]-i \alpha \sigma_{y},
$$

while that for the diffusion is

$$
\hat{B}_{\mathrm{c}}(t)=\sqrt{\gamma}\left[\sigma-\left\langle\sigma_{x}\right\rangle_{\mathrm{c}}(t) / 2\right] .
$$

Both of these operators are conditioned in that they depend on the system average

$$
\left\langle\sigma_{x}\right\rangle_{\mathrm{c}}(t)=\left\langle\psi_{\mathrm{c}}(t)\left|\sigma_{x}\right| \psi_{\mathrm{c}}(t)\right\rangle .
$$

As stated above, on average the system still obeys the master equation (2.1). This is easiest to see from the stochastic master equation (SME), which allows for impure initial conditions. The SME can be derived from the SSE by constructing

$$
\begin{aligned}
d\left(\left|\psi_{\mathrm{c}}\right\rangle\left\langle\psi_{\mathrm{c}}\right|\right)= & \left(d\left|\psi_{\mathrm{c}}\right\rangle\right)\left\langle\psi_{\mathrm{c}}|+| \psi_{\mathrm{c}}\right\rangle\left(d\left\langle\psi_{\mathrm{c}}\right|\right) \\
& +\left(d\left|\psi_{\mathrm{c}}\right\rangle\right)\left(d\left\langle\psi_{\mathrm{c}}\right|\right),
\end{aligned}
$$

using the Itô rule (2.12), and then identifying $\left|\psi_{\mathrm{c}}\right\rangle\left\langle\psi_{\mathrm{c}}\right|$ with $\rho_{\mathrm{c}}$. The result is

$$
d \rho_{\mathrm{c}}=d t \gamma \mathcal{D}[\sigma] \rho_{\mathrm{c}}-i d t \alpha\left[\sigma_{y}, \rho_{\mathrm{c}}\right]+d W(t) \sqrt{\gamma} \mathcal{H}[\sigma] \rho_{\mathrm{c}},
$$

where $\mathcal{H}[A] B \equiv A B+B A^{\dagger}-\operatorname{Tr}\left[A B+B A^{\dagger}\right] B$. Although this has been derived assuming pure initial conditions, it is valid for any initial conditions [33]. This is also an Itô equation, which means the evolution for the ensemble average state matrix

$$
\rho(t)=\mathrm{E}\left[\rho_{\mathrm{c}}(t)\right]
$$

is found simply by averaging over the photocurrent noise term by using Eq. (2.13). This procedure yields the original master equation (2.1) again. The term "quantum trajectory" can be applied to any stochastic conditioned evolution of the system, be it described by a SSE or SME.

\section{MARKOVIAN FEEDBACK STABILIZATION}

We now include feedback onto the amplitude of the driving on the atom, proportional to the homodyne photocurrent, as done by HMH. This is as shown in Fig. 1, where the driving field passes through an electro-optic amplitude modulator controlled by the photocurrent, yielding a field proportional to $\alpha+\lambda I(t)$. This means that the feedback can be described by the Hamiltonian

$$
H_{\mathrm{fb}}=\lambda \sigma_{y} I(t) .
$$

In this section we are assuming instantaneous feedback, while feedback with time delay will be considered in the next section.

Since the homodyne photocurrent 2.10 ) is defined in terms of system averages and the noise $d W(t)$, the SSE including feedback can still be written as an equation of the form (2.15). The effect of the feedback Hamiltonian can be shown [11,25] to change the drift and diffusion operators to

$$
\begin{aligned}
\hat{A}_{\mathrm{c}}(t)= & \frac{\gamma}{2}\left[-\sigma^{\dagger} \sigma+\left\langle\sigma_{x}\right\rangle_{\mathrm{c}}(t) \sigma-\left\langle\sigma_{x}\right\rangle_{\mathrm{c}}^{2}(t) / 4\right]-i \alpha \sigma_{y} \\
& +\lambda \sqrt{\gamma}\left[-i\left\langle\sigma_{x}\right\rangle_{\mathrm{c}}(t) \sigma_{y}-2 \sigma^{\dagger} \sigma\right]-\lambda^{2} / 2 \\
\hat{B}_{\mathrm{c}}(t)= & \sqrt{\gamma}\left[\sigma-\left\langle\sigma_{x}\right\rangle_{\mathrm{c}}(t) / 2\right]-i \lambda \sigma_{y} .
\end{aligned}
$$

Say we wish to stabilize the pure state with Bloch angle $\theta_{0}$, as defined in Eq. (2.6), with $r=1$ of course. In terms of the ground and excited states, this state is

$$
\left|\theta_{0}\right\rangle=\cos \frac{\theta_{0}}{2}|e\rangle+\sin \frac{\theta_{0}}{2}|g\rangle .
$$

Now for this state to be stabilized we must have

$$
\left[\hat{A}_{\mathrm{c}}(t) d t+\hat{B}_{\mathrm{c}}(t) d W(t)\right]\left|\theta_{0}\right\rangle \propto\left|\theta_{0}\right\rangle .
$$

We cannot say the left-hand-side should equal zero because a change in the overall phase still leaves the physical state unchanged. However, we can work with this equation, and simplify it by dropping all terms proportional to the identity operator in $\hat{A}_{\mathrm{c}}(t)$ and $\hat{B}_{\mathrm{c}}(t)$. We can also demand that it be satisfied for the deterministic and noise terms separately, because $d W(t)$ can take any value. This gives the two equations

$$
\begin{aligned}
& \left(\sqrt{\gamma} \sigma-i \lambda \sigma_{y}\right)\left|\theta_{0}\right\rangle \propto\left|\theta_{0}\right\rangle, \\
& {\left[\gamma\left(-\sigma^{\dagger} \sigma+\sin \theta_{0} \sigma\right)-i 2 \alpha \sigma_{y}\right.} \\
& \left.+\lambda \sqrt{\gamma}\left(-i \sin \theta_{0} \sigma_{y} / 2-\sigma^{\dagger} \sigma\right)\right]\left|\theta_{0}\right\rangle \propto\left|\theta_{0}\right\rangle,
\end{aligned}
$$

where we have put $\left\langle\sigma_{x}\right\rangle_{\mathrm{c}}(t)$ equal to $\sin \theta_{0}$, its value for the state $\left|\theta_{0}\right\rangle$.

Solving the first equation easily yields the condition

$$
\lambda=-\frac{\sqrt{\gamma}}{2}\left(1+\cos \theta_{0}\right) .
$$

Substituting this into the second equation gives, after some trigonometric manipulation, the second condition

$$
\alpha=\frac{\gamma}{4} \sin \theta_{0} \cos \theta_{0} .
$$

These set of driving and feedback amplitude can stabilize the system in any particular state in the Bloch sphere (except those on the equator; see below) for unit-detection efficiency and Markovian feedback. These features are illustrated in Fig. 2 as a full circle (minus the points on the equator) which is the locus for the equations with optimal driving and feedback. This is contrary to the 
conclusion of HMH 24, based on a linearized stability analysis, that "long term stability of ... inverted states [i.e. states in the upper half plane] cannot be achieved."

Once again, it is convenient to construct the stochastic master equation (SME). This can be derived from the $\mathrm{SSE}$ in the same way as before [Eq. (2.19)]. The result is 11,25

$$
\begin{aligned}
d \rho_{\mathrm{c}}= & d t \gamma \mathcal{D}[\sigma] \rho_{\mathrm{c}}-i d t \alpha\left[\sigma_{y}, \rho_{\mathrm{c}}\right] \\
& -i d t \lambda\left[\sigma_{y}, \sigma \rho_{\mathrm{c}}+\rho_{\mathrm{c}} \sigma^{\dagger}\right]+d t\left(\lambda^{2} / \gamma\right) \mathcal{D}\left[\sigma_{y}\right] \rho_{\mathrm{c}} \\
& +d W(t) \mathcal{H}\left[\sqrt{\gamma} \sigma-i \lambda \sigma_{y}\right] \rho_{\mathrm{c}}
\end{aligned}
$$

Also as before, this is an Itô stochastic equation, which means that the ensemble average can be found simply by dropping the stochastic terms. This time, the result is not the original master equation, but rather the feedbackmodified master equation

$$
\dot{\rho}=-i\left[\alpha \sigma_{y}, \rho\right]+\mathcal{D}\left[\sqrt{\gamma} \sigma-i \lambda \sigma_{y}\right] \rho \equiv \mathcal{L} \rho .
$$

Here we have put the Liouvillian superoperator $\mathcal{L}$ in a manifestly Lindblad form.

It can be shown that the pure state $\rho=\left|\theta_{0}\right\rangle\left\langle\theta_{0}\right|$ is a stationary solution of this master equation, as expected. However, if and only if $\theta_{0}= \pm \pi / 2$, it is not a stable solution. That is because the master equation for $\theta_{0}= \pm \pi / 2$ (it is the same for both cases) has more than one null eigenvalue, and any mixture of the two equatorial states will be a stationary solution. This means that states on the equator, which are equal superpositions of excited and ground states, cannot be well-protected against decoherence. We will return to this in Sec. IV C.

\section{NON-MARKOVIAN FEEDBACK}

\section{A. Non-Markovian feedback SME}

Now we consider the case of non-Markovian feedback. This would occur experimentally unless the response of the feedback apparatus is flat in frequency space over a bandwidth much larger than any relevant system rate. For simplicity, we consider the case of just having a time delay $\tau$. That is, we take the feedback Hamiltonian to be

$$
H_{\mathrm{fb}}=\lambda \sigma_{y} I(t-\tau) .
$$

The Stochastic Master Equation (SME ) of the total conditioned evolution of the system for $\tau$ finite is given by [11, 12

$$
\begin{aligned}
d \rho_{\mathrm{c}}= & d t \gamma \mathcal{D}[\sigma] \rho_{\mathrm{c}}-i d t \alpha\left[\sigma_{y}, \rho_{\mathrm{c}}\right]+d W(t) \sqrt{\gamma} \mathcal{H}[\sigma] \rho_{\mathrm{c}} \\
& -\lambda d t I(t-\tau) i\left[\sigma_{y}, \rho_{\mathrm{c}}\right]-d t\left(\lambda^{2} / 2\right)\left[\sigma_{y},\left[\sigma_{y}, \rho_{\mathrm{c}}\right]\right]
\end{aligned}
$$

Here $I(s)$ is still given by Eq. (2.14), so that the noise $d W(s)$ appears twice in Eq. (4.2), with two different time arguments, $s=t$ and $s=t-\tau$.
In Eq. (4.2) it is no longer possible simply to set the noise $d W$ equal to its expectation value of zero to obtain a deterministic equation. That is because the $d W(t-\tau)$ appearing in the feedback through $I(t-\tau)$ is correlated with the system state, since it already conditioned it through the measurement term at time $t-\tau$. Also, the atomic dynamics are nonlinear, so there is no option but to solve Eq. (4.2) numerically. The most convenient way to treat the stochastic dynamics is, in general, to use stochastic Bloch Equations (SBE). These are simply the stochastic equations for the conditioned Bloch vector, defined by

$$
\rho_{\mathrm{c}}=\frac{1}{2}\left(I+x_{\mathrm{c}} \sigma_{x}+y_{\mathrm{c}} \sigma_{y}+z_{\mathrm{c}} \sigma_{z}\right) .
$$

The SBE corresponding to Eq. (4.2) are

$$
\begin{aligned}
\left(\begin{array}{l}
d x_{\mathrm{c}} \\
d y_{\mathrm{c}} \\
d z_{\mathrm{c}}
\end{array}\right)= & \left(\begin{array}{ccc}
-\gamma / 2-2 \lambda^{2} & 0 & 2 \alpha+2 \lambda I_{-\tau} \\
0 & -\gamma / 2 & 0 \\
-2 \alpha-2 \lambda I_{-\tau} & 0 & -\gamma-2 \lambda^{2}
\end{array}\right) \\
& \times\left(\begin{array}{c}
x_{\mathrm{c}} \\
y_{\mathrm{c}} \\
z_{\mathrm{c}}
\end{array}\right) d t-d t\left(\begin{array}{c}
0 \\
0 \\
-\gamma
\end{array}\right) \\
& +\sqrt{\gamma} d W(t)\left(\begin{array}{c}
1-x_{\mathrm{c}}^{2}+z_{\mathrm{c}} \\
-x_{\mathrm{c}} y_{\mathrm{c}} \\
-x_{\mathrm{c}}-x_{\mathrm{c}} z_{\mathrm{c}}
\end{array}\right)
\end{aligned}
$$

Here we have used $I_{-\tau}$ as short-hand for $I(t-\tau)$.

We can use these equations to perform the numerical simulation of the non-Markovian process. In the numerical simulation, we have not optimized the driving and feedback amplitude to achieve the best possible result for a given time delay $\tau$. This is as opposed to the Markovian case with inefficient detection where we optimized for every $\eta$. The difference is because in the present case the optimization problem is considerable more difficult. However, since we expect that the feedback mechanism will only work effectively with a small feedback delay time, it is thus convenient and reasonable to use the same set of driving and feedback amplitude as in the Markovian case, namely

$$
\begin{aligned}
& \lambda=-\frac{\sqrt{\gamma}}{2}\left(1+\cos \theta_{0}\right), \\
& \alpha=\frac{\gamma}{4} \sin \theta_{0} \cos \theta_{0} .
\end{aligned}
$$

\section{B. The effect on stability and purity}

Note from Eq. (4.4) that $y_{\mathrm{c}}=0$ is a stationary solution. Assuming this value for $y_{\mathrm{c}}$, we can again use the polar coordinates $\theta$ and $r$ as in Eq. (2.6). Their equations of motion are found from

$$
\begin{array}{r}
\theta+d \theta=\tan ^{-1}\left(\frac{x_{\mathrm{c}}+d x_{\mathrm{c}}}{z_{\mathrm{c}}+d z_{\mathrm{c}}}\right), \\
r+d r=\sqrt{\left(x_{\mathrm{c}}+d x_{\mathrm{c}}\right)^{2}+\left(z_{\mathrm{c}}+d z_{\mathrm{c}}\right)^{2}} .
\end{array}
$$


Using the Taylor expansion and Itô rules, and assuming that the state is initially pure $(r=1)$ we find

$$
d r=0
$$

and

$$
\begin{aligned}
d \theta= & {\left[2 \alpha+2 \sqrt{\gamma} \lambda I(t-\tau)+\frac{\gamma}{2}(2+\cos \theta) \sin \theta\right] d t } \\
& +\sqrt{\gamma}(1+\cos \theta) d W(t),
\end{aligned}
$$

where

$$
I(t-\tau) d t=\sqrt{\gamma} \sin \theta(t-\tau) d t+d W(t-\tau) .
$$

That is, the state remains pure and the simulation reduces to the single non-Markovian stochastic differential equation (4.10) which is readily solved in Matlab.

The two plots in Fig. 3 are the typical quantum trajectories in Bloch space for $\theta_{0}=\pi / 6$, starting at the ground state. The main plot with more dramatic fluctuations is the trajectory via the path through the ground state with feedback time delay $\tau=0.02 \gamma^{-1}$, while the plot with an inset into this figure is the one for feedback with no time delay and the trajectory is via the path through the excited state. Clearly from these two trajectories, there are two ways for the system to reach a desired state of $\theta=\theta_{0}$. As shown in this figure, the trajectory of the main plot with $\tau=0.02 \gamma^{-1}$ continues to evolve stochastically after the transients have died away. The parameter $\theta$ wanders around the desired the pure state $\theta_{0}=\pi / 6$ even when the system is in steady state. By contrast, that of the inset for Markovian feedback stops precisely at the desired pure steady state with no fluctuations persistent. Moreover, we find that the longer the feedback delay time is, the more dramatic the fluctuations are. Therefore, the longer time delay will result in more randomness in the steady state quantum trajectory. This indicates that the stability of the single trajectory is reduced by the effect of non-zero feedback delay time.

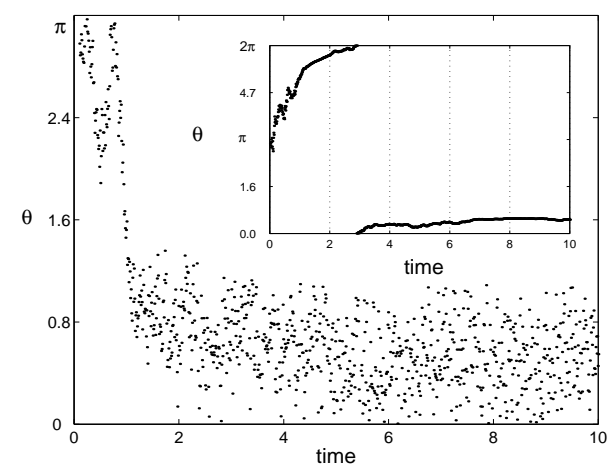

FIG. 3. Typical quantum trajectories for $\theta$ (representing the conditioned state), for $t \in\left[0,10 \gamma^{-1}\right]$ and $\theta_{0}=\pi / 6$, starting at the ground state. Note that the single trajectory can go either via the ground state or the excited state to get to $\theta_{0}=\pi / 6$. The main plot with a more dramatic fluctuations is for feedback with a large time $\tau=0.02 \gamma^{-1}$, while the inset is for feedback with no time delay.
Another typical trajectory, this time for $\theta_{0}=0$, is shown in Fig. 4 in Bloch space for $t \in\left[0,100 \gamma^{-1}\right]$ with a feedback delay time $\tau=0.02 \gamma^{-1}$, again starting at the ground state. Firstly, to consider the stability of the single trajectory, we see that the initial evolution is erratic, then on a time scale of a few $\gamma^{-1}$ the system relaxes towards the desired state $\theta_{0}=0$. As in the previous case, the fluctuations are still persistent even in steady state and there is considerable variation around the desired angle of $\theta_{0}=0$. Secondly, to consider the purity of the single trajectory, we can see from this plot that in a single trajectory the system state is always pure; it lives on the surface of the Bloch sphere. However, an ensemble (or time) average of the system state will put it inside the Bloch sphere. The time-averaged state from this trajectory (ignoring transients) is represented by the single star dot underneath the point $\theta_{0}=0$. The larger the fluctuations around $\theta_{0}$, the closer this point would be towards the center of the sphere and the lower the purity of the average state. Therefore, we can see that the purity of a single trajectory is not be affected by the non-Markovian process while the purity of the average state is. Rather, the purity of the average state reflects the stability of the individual trajectory.

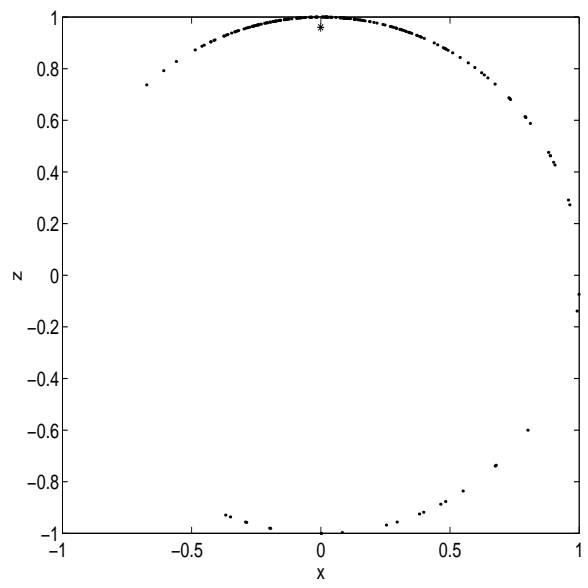

FIG. 4. Typical single quantum trajectories in Bloch space for $t \in\left[0,100 \gamma^{-1}\right]$ with $\tau=0.02 \gamma^{-1}$ and $\theta_{0}=0$, starting at the ground state. Note that the single star dot underneath the single trajectory path indicates the loss of purity of the average state, while the single trajectory evolves stochastically along the outside full circle which is the locus of pure states.

Fig. 5 is the plot of the locus of the time-averaged states in Bloch space for three different feedback delay times $\tau=0,0.02 \gamma^{-1}$, and $0.2 \gamma^{-1}$. For each time delay we plot many points, each corresponding to a different value of $\theta_{0}$, the angle at which the Markovian feedback would stabilize the state. We have deliberately symmetrized this plot by combining the simulations of positive and negative $\theta$. 
A number of points are worth noting. First, and most obviously, the degree of purity (measured by the $r$, the distance from the origin) decreases with $\tau$. Second, the gap at the equator for $\tau=0$ quickly widens for larger delay time $\tau$, so that the purity of the time-averaged states with $\theta$ close to $\pi / 2$ is decreased, which indicates that the states near the equator can not be well-protected against decoherence. Third, the purity of the time-averaged states in the upper half of the Bloch sphere is affected much more by the increase of delay time $\tau$ than those in the lower half. Since for very long time delay the feedback could not be effective, and since the stationary states with no feedback are confined to the lower half of the Bloch sphere, this is perhaps not surprising. Fourth, the bottom half curves for the larger delay time are closer to the ground state even than the no-feedback curve of Fig. 2. This is due to the fact that we have not optimized the driving and feedback amplitude to search out the best possible pure state. With a proper choice of driving and feedback amplitude, the curves should always lie between the no-feedback ellipse of Fig. 2 and the $r=1$ pure state circle.

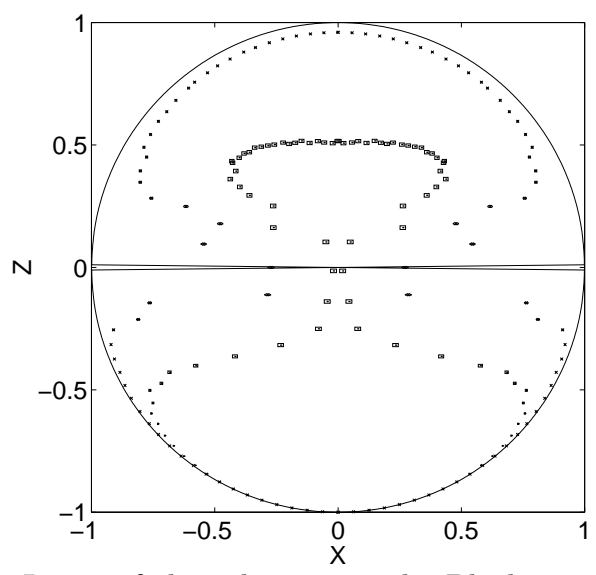

FIG. 5. Locus of the solutions to the Bloch equations for feedback with different values of delay time $\tau$. From the outside in, we have $\tau=0,0.02 \gamma^{-1}$, and $0.2 \gamma^{-1}$. The plot with $\tau=0$ is an analytical solution, and the others with error bars are from numerical simulations. The simulation time for each time-averaged state is $10^{4} \gamma^{-1}$, giving an effective sample size of order $10^{4}$, since $\gamma^{-1}$ is of order the correlation time of the system.

All of these features (except the last, which was avoided through proper optimization) also arose from feedback with non-unit-efficiency detection, as discussed in our previous paper [22]. Both non-Markovian feedback and non-unit-efficiency detection decrease the degree of stability of the system and thus to limit the capability of feedback decoherence control. The difference is that with non-unit efficiency detection not only the average, but also the individual conditioned state of the system is impure.

\section{Exception: the equatorial states}

As mentioned in Sec. III, the story for trying to stabilize the equatorial states $\theta_{0}= \pm \pi / 2$ is special. First, consider the Markovian case. The SBEs for the equatorial states are

$$
\begin{aligned}
\left(\begin{array}{l}
d x_{\mathrm{c}} \\
d y_{\mathrm{c}} \\
d z_{\mathrm{c}}
\end{array}\right)= & d t\left(\begin{array}{ccc}
0 & 0 & 0 \\
0 & -\gamma / 2 & 0 \\
0 & 0 & -\gamma / 2
\end{array}\right)\left(\begin{array}{l}
x_{\mathrm{c}} \\
y_{\mathrm{c}} \\
z_{\mathrm{c}}
\end{array}\right) \\
& +\sqrt{\gamma} d W(t)\left(\begin{array}{c}
1-x_{\mathrm{c}}^{2} \\
-x_{\mathrm{c}} y_{\mathrm{c}} \\
-x_{\mathrm{c}} z_{\mathrm{c}}
\end{array}\right) .
\end{aligned}
$$

Both $z_{c}$ and $y_{c}$ will decay to zero (as required for $|\theta|=$ $\pi / 2$ ), and their noise terms vanish at that point. By contrast, the equation for $x_{\mathrm{c}}$ is independent of the others, and is purely stochastic:

$$
d x_{\mathrm{c}}=\sqrt{\gamma} d W(t)\left(1-x_{\mathrm{c}}^{2}\right)
$$

Clearly the equatorial pure states with $x_{\mathrm{c}}= \pm 1$ are stationary solutions to this problem. Also, the system will tend to one of these states. But it is also clear that $x_{\mathrm{c}}$ has no preference to go to either of these states. Hence they are not stable. The ensemble average $x$ is unchanging under this evolution. Thus a perturbation which moves the state from $x_{\mathrm{c}}=1$ to $x_{\mathrm{c}}=1-\epsilon$ say, will result in a proportion $\epsilon / 2$ of the states ending up at $x_{\mathrm{c}}=-1$, and a proportion $1-\epsilon / 2$ ending up at $x_{\mathrm{c}}=1$.

The above discussion all refers to the Markovian case. Having a time delay will introduce extra noise in the system, as seen in the preceding section. This is a sort of perturbation that will disturb the state of the system. Thus we expect that with a time delay, even if the system is close to one of the fixed states $x_{\mathrm{c}}= \pm 1$, it will not stay there. Rather, we expect that it will at some point randomly switch to being close to the other fixed point. This is indeed what we observe, as we illustrate by showing a typical trajectory for $\theta_{0}=\frac{\pi}{2}$ in Fig. 6, again starting from ground state. On a time scale of a few $\gamma^{-1}$ the system reaches the region of the no-delay fixed point $x_{\mathrm{c}}=1$, on one side of the equator. Then at a later time, about $5 \gamma^{-1}$ here, it stochastically hops to the other side of the Bloch sphere (going through the ground state again), ending up near the other fixed point with $x_{\mathrm{c}}=-1$. 


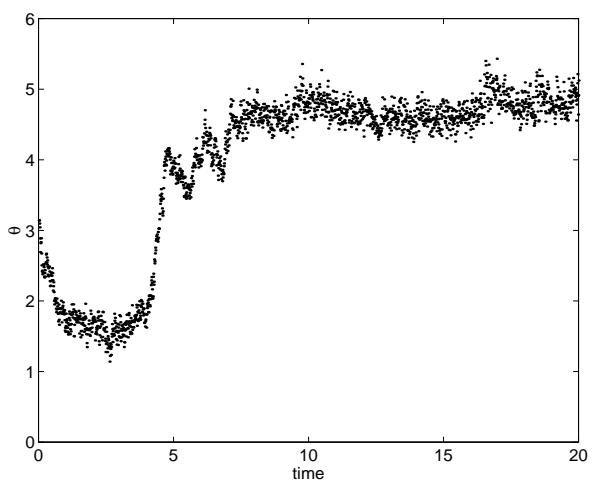

FIG. 6. Typical quantum trajectories for $\theta_{0}=\pi / 2$ with driving amplitude $\alpha=0$, feedback amplitude $\lambda=-\sqrt{\gamma} / 2$ and delay time $\tau=0.02 \gamma^{-1}$, shown by $\theta$ (dots) as functions of time $t \in\left[0,20 \gamma^{-1}\right]$. This plot shows that the system has no preference to go to either equatorial point $x_{\mathrm{c}}=1(\theta=\pi / 2)$ or $x_{\mathrm{c}}=-1(\theta=3 \pi / 2)$.

\section{Analytical solution}

As we mentioned in the introduction, the excited state $\theta_{0}=0$ is the only state where we can get both analytical and numerical solutions to the non-Markovian feedback process. In this subsection, we will derive the analytical solution of the excited state and compare it with the numerical one.

For short time delay we expect that (except for states near the equator), $\theta$ will stay near the desired $\theta_{0}$. Thus, it makes sense to define $\delta \theta=\theta-\theta_{0}$ and do a perturbative expansion for small $\delta \theta$ in Eq. (4.5), Eq. (4.6) and Eq. (4.10). This gives the Itô equation

$$
\begin{aligned}
\delta \dot{\theta(t)=} & -\gamma\left(1+\cos \theta_{0}\right) \cos \theta_{0} \delta \theta(t-\tau) \\
& +\gamma\left(\cos \theta_{0}+\frac{1}{2} \cos 2 \theta_{0}\right) \delta \theta(t)-\sqrt{\gamma} \sin \theta_{0} \delta \theta \xi(t) \\
& -\sqrt{\gamma}\left(1+\cos \theta_{0}\right)[\xi(t-\tau)-\xi(t)] .
\end{aligned}
$$

Now the purity of the average state can be defined as

$$
P=2 \operatorname{Tr}\left[\rho^{2}\right]-1=r^{2}=\left\langle x_{c}\right\rangle^{2}+\left\langle y_{c}\right\rangle^{2}+\left\langle z_{c}\right\rangle^{2} .
$$

Using $y_{c}=0, x_{c}=\sin \theta, z_{c}=\cos \theta$ and assuming $\langle\delta \theta\rangle=0$ yields, to leading order,

$$
P \simeq 1-\left\langle(\delta \theta)^{2}\right\rangle .
$$

Thus if we can find an expression for $\left\langle(\delta \theta)^{2}\right\rangle$ we can find the purity of the average state. The decrease in purity due to fluctuations in $\delta \theta$ can be seen directly in Fig. 4, for the case $\theta_{0}=0$.

Inspecting Eq. (4.14) we can see that it is solvable only if $\sin \theta_{0}=0$ so that the multiplicative term $\delta \theta \xi(t)$ vanishes. This will be the case only when one is trying to put the atom in the excited state $\theta_{0}=0$, or the ground state $\theta_{0}=\pi$. The latter is trivial, as it is achieved exactly with no feedback or driving. The former is the interesting case, and with $\theta_{0}=0$, Eq. (4.14) becomes $\dot{\delta} \theta(t)=-2 \gamma \delta \theta(t-\tau)+\frac{3 \gamma}{2} \delta \theta(t)-2 \sqrt{\gamma}[\xi(t-\tau)-\xi(t)]$,

We show in the appendix that we can solve this equation exactly in Fourier space, and use the result to evaluate $\left\langle(\delta \theta)^{2}\right\rangle$ approximately, in the limit $\tau \ll \gamma^{-1}$. The result is

$$
P=1-4 \gamma \tau \text {. }
$$

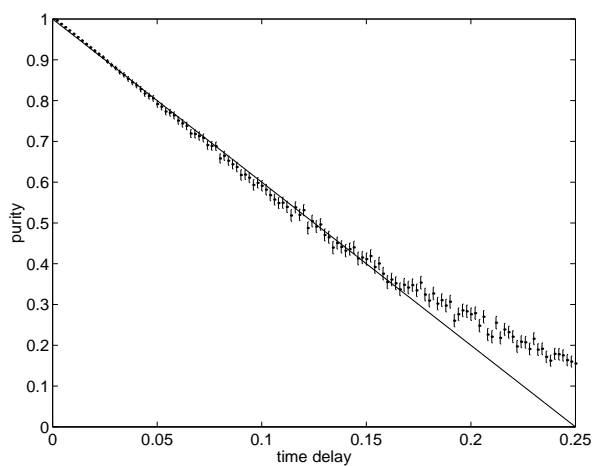

FIG. 7. Plot of the purity against time delay for the atom near the excited state. The solid line is the theoretical result while the dotted line with error bars is the numerical one. The analytical result agrees very well with the numerical one when feedback delay time is less than $0.17 \gamma^{-1}$.

We plot in Fig. 7 the purity against delay time for the atom with $\theta_{0}=0$, according to the analytical approximation (4.18) and from numerical simulations. Clearly the analytical result agrees very well with the numerical one when the feedback delay time is less than about $0.17 \gamma^{-1}$. When the feedback delay time is larger than $0.17 \gamma^{-1}$, a significant difference appears, and increases as the feedback delay time becomes larger. However, as we can see from Eq. (4.18), the feedback delay time must be less than $0.25 \gamma^{-1}$ in Eq. (4.18) in order to satisfy $P>0$. Therefore for the atom near the excited state, our analytical solution is a good approximation for a surprisingly large delay time $\tau$.

\section{DISCUSSION}

In this paper, we have given a rigorous analysis of an anti-decoherence feedback scheme in a two-level atom in the limit where the feedback time delay is significant. We use numerical simulations to determine the influence of the non-Markovicity on the effectiveness of the feedback. We find that, unlike the case for Markovian feedback, it is not possible to stabilize the atom in a fixed pure state (except the ground state which is trivially pure by setting the driving and feedback to zero). Although the conditioned system state always remains pure (since we are 
assuming perfect detection) it does not remain stable but continues to wander around the desired fixed pure state. Thus the ensemble-averaged state (which is the timeaveraged state in steady state) is not pure, but mixed. As expected, the longer the feedback delay time is, the more dramatic the fluctuations are and consequently the less pure the average state is.

We find that the purity of states in the upper half of the Bloch sphere is affected much more by feedback delay than those in the lower half. This is not unexpected, as with no feedback states in upper half cannot be produced at all. Also, the purity of states near the equator of the Bloch sphere is very much affected. This is because the feedback algorithm for stabilizing states on the equator cannot distinguish between diametrically opposite points on the equator. Because delayed feedback cannot perfectly cancel the measurement back-action noise, trying to stabilize at a point on the equator will give a conditioned state which flips at random from one side to the other, on a time scale that decreases as the time delay increases. The unconditioned (or time-averaged) state is subsequently almost completely mixed. All of these features are qualitatively similar to the results obtained for no time delay but with inefficient detection in Ref. 22. The major consequence is that states which are nearequal superpositions of excited and ground states cannot be well-protected against decoherence.

There is one case in which it is possible to obtain analytical results, namely when one tries to stabilize the atom in the excited state. Our approximate analytical result that the purity (the square of the Bloch radius) of the average state is given by $P=1-4 \gamma \tau$ (where $\tau$ is the delay and $\gamma$ the spontaneous emission rate) agrees very well with our numerical results even for quite large $\tau$, up to $0.17 \gamma^{-1}$. This shows the usefulness of the quantum trajectory approach to feedback. Not only is it the only practical way to treat a non-Markovian problem, it can also yield analytical solutions in some cases. Other approaches to non-Markovian feedback can only be used for systems with linear dynamics, and it is not clear that a linearization procedure would work even for the special case of stabilizing near the excited state.

The control of decoherence has become an important topic in recent years in many related research areas. In quantum computation decoherence is a main limiting factor. In a quantum computer information is not stored as bits, but rather as qubits [36]. A qubit is a quantum system with a two dimensional Hilbert space, such as a twolevel atom. The logical basis states are two orthogonal basis states, $|0\rangle,|1\rangle$, but the qubit may be in a superposition of these states. A quantum computer would consist of a large number $N$ of qubits, and could exist as an arbitrary state in the $2^{N}$-dimensional Hilbert space. If any individual qubit undergoes decoherence, such as spontaneous emission, the quantum computation is destroyed. Furthermore this decoherence rate scales linearly with the number of two-level systems. If quantum computation is to become a reality a means must be found to control such decoherence. Sophisticated quantum error correction methods have been developed to detect and control arbitrary amplitude and phase errors [37. While our decoherence-control scheme is by no means as general as error correction, it may be of use for maintaining qubits in a particular pure state until required.

We close by saying a few words about experimental realizability. The results of this work shows that a time delay $\tau$ is not fatal to controlling decoherence even in a nonlinear system like the two-level atom. The purity of the state will, in most cases, remain close to unity as long as $\tau$ is much less than the atomic lifetime $\gamma^{-1}$. This is feasible with very fast electronics. The greater difficulty is with obtaining high efficiency in detection. Collecting a large proportion of the light emitted by an atom in free space is very difficult. It is much easier to collect the light emitted from a cavity, as this propagates in one direction. Therefore, the most likely scenario for realizing the scheme would be in the context of cavity QED [38]. If a two-level atom is strongly coupled $(g)$ to a single cavity mode, which is strongly damped $(\kappa)$, the combined system acts like an effective two-level atom. The output beam of the cavity is effectively the spontaneous emission of the atom. Then, given that the time delay in the feedback loop is relatively small compared to the effective atomic lifetime $\sim \kappa / g^{2}$ ), we can control the decoherence of the cavity QED system.

\section{ACKNOWLEDGMENTS}

This work has been supported by the Australian Research Council, the University of Queensland, and the Department of Employment, Education and Training, Australia. JW would like to thank Prof. H. Carmichael and A/Prof. Z. Ficek for useful discussions.

\section{APPENDIX}

For the atom in the excited state, the SBE in the time domain is given by

$$
\begin{aligned}
\dot{\delta \theta}(t)= & -2 \gamma \delta \theta(t-\tau)+\frac{3 \gamma}{2} \delta \theta(t) \\
& -2 \sqrt{\gamma}[\xi(t-\tau)-\xi(t)] .
\end{aligned}
$$

Applying the Fourier transformation, we can solve the $\mathrm{SBE}$ in the frequency domain:

$$
\tilde{\delta \theta}(\omega)=\frac{\sqrt{\gamma}\left(e^{i \omega \tau}-1\right) \tilde{\xi}(\omega)}{\left[\gamma\left(\frac{3}{2}-2 e^{i \omega \tau}\right)+i \omega\right]}
$$

Applying the inverse Fourier transformation to go back to the time domain, we get

$$
\delta \theta(t)=\frac{1}{2 \pi} \int_{-\infty}^{\infty} d \omega e^{-i \omega t} \frac{2 \sqrt{\gamma}\left(e^{i \omega \tau}-1\right)}{\gamma\left(\frac{3}{2}-2 e^{i \omega \tau}\right)+i \omega} \xi(\omega) .
$$


Since $\left\langle\xi(\omega) \xi\left(\omega^{\prime}\right)\right\rangle=2 \pi \delta\left(\omega+\omega^{\prime}\right)$, we find

$$
\begin{aligned}
\left\langle\delta \theta^{2}(t)\right\rangle & =\frac{1}{2 \pi} \int_{-\infty}^{\infty} \frac{4 \gamma(2-2 \cos \omega \tau) d \omega}{\gamma^{2}\left(\frac{3}{2}-2 \cos \omega \tau\right)^{2}+(\omega-2 \gamma \sin \omega \tau)^{2}} \\
& =\frac{1}{\pi \gamma} \int_{0}^{\infty} \frac{16 \sin ^{2}(\omega \tau / 2) d \omega}{\left(\frac{3}{2}-2 \cos \omega \tau\right)^{2}+(\omega / \gamma-2 \sin \omega \tau)^{2}} .
\end{aligned}
$$

The above integral is too complicated to be useful. Also, we are really only interested in the limit $\tau \ll \gamma^{-1}$, since that is the limit in which $\delta \theta$ is expected to be small so that the approximations leading to Eq. (6.1) will be valid. With this in mind, consider a parameter $c$ such that $\gamma \ll c \ll \tau^{-1}$, and $c^{3} \tau \ll \gamma^{2}$. Then split the $\omega$ integration up as $\int_{0}^{\infty}=\int_{0}^{c}+\int_{c}^{\infty}$. When $\omega<c$, we have $16 \sin ^{2} \omega \tau / 2<(2 \omega \tau)^{2} \ll 1$ in the numerator, while the denominator is greater than or equal to $\frac{1}{4}$. Therefore the integration from 0 to $c$ is bounded above by $16 \tau^{2} c^{3} / 3$. On the other hand, when $\omega>c$, we have $\omega \gg \gamma$ so that $\omega / \gamma$ is much greater than all the other terms in the denominator. Therefore we can approximate the integration in this region as

$$
\int_{c}^{\infty} d \omega \frac{16 \gamma^{2} \sin ^{2}(\omega \tau / 2)}{\omega^{2}}
$$

which, since $c \ll \tau^{-1}$, can be further approximated as

$$
\int_{0}^{\infty} d \omega \frac{16 \gamma^{2} \sin ^{2}(\omega \tau / 2)}{\omega^{2}}=\gamma^{2} 4 \pi \tau
$$

Since by assumption $c^{3} \tau \ll \gamma^{2}$, this integral is always much greater than that from 0 to $c$. We thus finally arrive at

$$
\left\langle\delta \theta^{2}(t)\right\rangle \simeq \frac{1}{\pi \gamma} \times \gamma^{2} 4 \pi \tau=4 \gamma \tau \ll 1 .
$$

[1] L. Diósi, N. Gisin, J. Halliwell and I. C. Percival, Phys. Rev. Lett.74, 203 (1995).

[2] N. Gisin and I. C. Percivl, J. Phys. A: Math. Gen 26, 2223 and 2245 (1993).

[3] P. Goetsch and R. Graham, Phys. Rev. A 50, 5242 (1994).

[4] T. P. Spiller and J. F. Ralph, Phys. Lett. A 194, 235 (1994).

[5] L. Viola, R. Onofrio and T. Calarco, Phys. Lett. A 229, 23 (1997).

[6] H. J. Carmichael, An Open Systems Approach to Quantum Optics (Springer-Verlag, Berlin, 1993).

[7] J. Dalibard, Y. Castin and K. Mølmer, Phys. Rev. Lett. 68, 580 (1992).

[8] C. W. Gardiner, A. S. Parkins, and P. Zoller, Phys. Rev. A 46, 4363 (1992).
[9] H. M. Wiseman and G. J. Milburn, Phys. Rev. A 47, 1652 (1993).

[10] H. M. Wiseman, Quant. Semiclass. Opt. 8, 205 (1996).

[11] H. M. Wiseman and G. J. Milburn, Phys. Rev. Lett. 70, 548 (1993).

[12] H. M. Wiseman, Phys. Rev. A 49, 2133 (1994).

[13] H. A. Haus and Y. Yamamoto, Phys. Rev. A 34, 270 (1986).

[14] Y. Yamamoto, N. Imoto and S. Machida, Phys. Rev. A 33, 3243 (1986).

[15] J. M. Shapiro et al, J. Opt. Soc. Am. B 4, 1604 (1987).

[16] J. G. Walker and E. Jakeman, Proc. Soc. Photo-Opt. Instrum. Eng. 492, 274 (1985).

[17] L. Plimak, Phys. Rev. A 50, 2120 (1994).

[18] Herschel Rabitz, Regina de Vivie-Riedle, Marcus Motzkus, Karl Kompa, Science, 288, 824 (2000).

[19] G. Raithel et al., in Cavity Quantum Electrodynamics, edited by aul R. Berman, Adv. At. Mol. Opt. Phys, Suppl. 2 (1994).

[20] D. J. Wineland, C. Monroe, W. M. Itano, D. Leibfried, B. E. King, and D. M. Meekhof, Journal of Research of the National Institute of Standards and Technology 103, 259 (1998).

[21] E. Buks, R. Schuster, M. Heiblum, D. Mahalu, and V. Umansky, Nature 391, 871 (1998).

[22] Jin Wang and H. M. Wiseman, "Feedback-stabilization of an arbitrary pure state of a two-level atom", to be published in Phys. Rev. A. quant-ph/0008003).

[23] J. P. Paz and W. H. Zurek, "Environment-Induced Decoherence and the Transition From Quantum to Classical", Lectures given at the 72nd Les Houches Summer School on Coherent Matter Waves, July-August 1999, (1999) (quant-ph/0010011).

[24] H. F. Hofmann, O. Hess, and G. Mahler, Optics Express 2, 339 (1998).

[25] H. M. Wiseman and G. J. Milburn, Phys. Rev. A 49, 1350 (1994).

[26] A. C. Doherty and K. Jacobs, Phys. Rev. A 60, 2700 (1999).

[27] V. Giovannetti, P. Tombesi, and D. Vitali, Phys. Rev. A, 60, 1549 (1999).

[28] A.C. Doherty, S. Habib, K. Jacobs, H. Mabuchi and S.M. Tan, Phys. Rev. A 62, 012105 (2000).

[29] M. W. Jack, M. J. Collett and D. F. Walls, Phys. Rev. A ,50, 2306 (1999).

[30] Lajos Diósi, and Walter T. Strunz Phys. Lett. A, 235, 569 (1997).

[31] Ting Yu, L. Diosi, N. Gisin, and W. T. Strunz, Phys. Rev. A, 60, 91 (2000).

[32] G. Lindblad, Commun. Math. Phys. 48, 199 (1976).

[33] H. M. Wiseman and G. J. Milburn, Phys. Rev. A 47, 642 (1993).

[34] C. W. Gardiner, Handbook of Stochastic Methods (Springer, Berlin, 1985).

[35] C. P. Williams and S. H. Clearwater, Explorations in Quantum Computing (Springer, New York, 1998).

[36] M. Nielsen and I. Chuang, "Quantum Computation and Quantum Information", Cambridge University Press, (Cambridge 2000). 
[37] C. M. Caves, in International Workshop on Macroscopic Quantum Tunneling and Coherence, Naples, Italy, June 10-13, 1998; to be published in Superconductivity (1998) (quant-ph/9811082).

[38] Q.A. Turchette et al. Phys. Rev. A 58, 4056 (1998). 